\title{
Appliqués d'une manière adéquate, les guides pratiques renforcent la prise en charge du patient
}

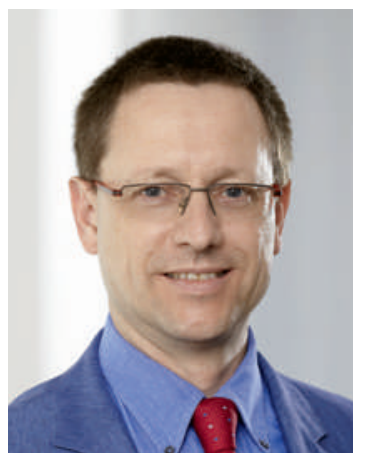

Les guides pratiques entrent également dans le domaine de compétence des médecins. Alors que les exigences publiées en 1999 par le Bulletin des médecins suisses sont pratiquement identiques à celles aujourd'hui en vigueur [1], notre tâche est de poursuivre ces travaux dans le respect des priorités médicales et déontologiques établies depuis longtemps déjà. Savoir si des recommandations pour la pratique clinique sont élaborées par une société de discipline ou reprises de standards reconnus au niveau international ne représente finalement qu'une petite différence. En revanche, il faut être conscient que le travail et la responsabilité à assumer sont du ressort de la société de discipline concernée. Grâce notamment aux indispensables possibilités de travail en réseau que lui offre l'Académie suisse pour la qualité en médecine (ASQM) et au savoir-faire scientifique de sa division Données, démographie et qualité, la FMH est en mesure de présenter dans ce numéro un aperçu de ce que la littérature spécialisée énonce aujourd'hui à ce sujet. Par ailleurs, le Forum Qualité de l'ASQM, point de rencontre entre les responsables qualité des sociétés de discipline, des sociétés cantonales de médecine et des associations faîtières, garantit le transfert de connais-

\section{Les guides pratiques contribuent à améliorer la prise en charge des patients.}

sances. En effet, les organisations qui ont déjà approfondi une question particulière peuvent faire partager leur savoir et leurs expériences. L'étape suivante à franchir reste toutefois celle de la standardisation. Même si du point de vue de la mise en œuvre ou de l'indication, certaines mesures médicales peuvent être standardisées, l'appréciation du patient est bien différente. Par deux fois, en 2008 et en 2012, les citoyennes et citoyens de ce pays ont plébiscité un modèle de prise en charge individuelle par le médecin de leur choix. Ce mandat politique rejoint notre expérience de médecin marquée par l'individualité du patient, notamment dans le cas de patients polymorbides qui peuvent subir les éventuelles contradictions de la standardisation et des guidelines. Notre pratique de la médecine nous amène à concevoir le patient comme un individu évoluant dans un environnement social donné et

\section{L'ASQM encourage et coordonne les échanges et le travail concernant la qualité.}

cette approche reste essentielle. Cependant, une prise en charge individuelle n'exclut en aucun cas le recours à des guides pratiques, notamment dans des situations qui relèvent de la routine quotidienne. Ce que d'un côté nous gagnons en efficacité grâce aux guides pratiques, nous pouvons le «réinvestir» en faveur des situations plus complexes de nos patients atteints de polymorbidité.

Les moyens financiers à disposition, la pénurie de praticiens mais aussi le vieillissement de notre société devraient nous inciter à réfléchir davantage à la question des guidelines. Nous devons également nous épauler mutuellement afin de pouvoir répartir la charge de travail qui en résultera. Les guides pratiques n'ouvrent-ils pas la voie à des trajectoires de patients pluridisciplinaires entre les secteurs ambulatoire et hospitalier? Je nous souhaite, à nous toutes et tous, la créativité nécessaire pour relever les défis à venir.

Dr Christoph Bosshard, membre du Comité central de la FMH, responsable du département Données, démographie et qualité

1 FMH. Directives pour les guides de pratique médicale. Bull Méd Suisses. 1999;80(19):581-3. 